\title{
On bifurcations of area-preserving and non-orientable maps with quadratic homoclinic tangencies *
}

\author{
A. Delshams ${ }^{\dagger}$, M. Gonchenko ${ }^{\ddagger}$ and S.V. Gonchenko ${ }^{\S}$ \\ † Departament de Matemàtica Aplicada I, Universitat Politècnica de Catalunya, Barcelona, Spain \\ ¥ Institut für Mathematik, Technische Universität Berlin, Germany \\ $\S$ Institute of Applied Mathematics and Cybernetics, Nizhny Novgorod University, Russia \\ amadeu.delshams@upc.edu, gonchenk@math.tu-berlin.de, gonchenko@pochta.ru
}

\begin{abstract}
We study bifurcations of non-orientable area-preserving maps with quadratic homoclinic tangencies. We study the case when the maps are given on non-orientable two-dimensional surfaces. We consider one and two parameter general unfoldings and establish results related to the emergence of elliptic periodic orbits.
\end{abstract}

\section{Introduction}

The main goal of the paper is the study of bifurcations of area-preserving maps (APMs) defined on non-orientable surfaces and possessing homoclinic tangencies.

For dissipative systems, the study of bifurcations of homoclinic tangencies is quite traditional and many results obtained here have a fundamental value for the theory of dynamical chaos. One of such results, known as theorem on cascade of periodic sinks (sources), goes back already to the paper [1] of Gavrilov and Shilnikov, see also [2, 3. Note that in this paper the general case was considered, when the initial two-dimensional diffeomorphism has a saddle fixed point $O$ with multipliers $\lambda$ and $\gamma$, where $0<|\lambda|<1<|\gamma|$ and the saddle value $\sigma \equiv|\lambda||\gamma|$ is not equal to 1 , and the invariant manifolds of $O$ are quadratically tangent at the points of some homoclinic orbit. In this case, bifurcations of the homoclinic tangency lead to the appearance of asymptotically stable (if $\sigma<1$ ) or completely unstable (if $\sigma>1$ ) periodic orbits. Moreover, in any one parameter general unfolding such orbits are observed for values of the parameter belonging to an infinite sequence (cascade) of intervals that do not mutually intersect and accumulate to the value of the parameter corresponding to the initial homoclinic tangency.

Concerning related results in the conservative case, we mention, above all, the well known result of S. Newhouse, 4], on the emergence of the so-called 1-elliptic periodic orbits (there is only one pair multipliers, $e^{i \varphi}$ and $e^{-i \varphi}$ with $\varphi \neq 0, \pi$, on the unit circle) under bifurcations of homoclinic

*This work has been partially supported by the Russian Scientific Foundation Grant 14-41-00044. Section 3 is carried out by the RSF-grant (project No.14-12-00811). The author SG has been partially supported by the by grants of RFBR No.13-01-00589, 13-01-97028-povolzhye and 14-01-00344. The authors AD and MG has been have been partially supported by the Spanish MINECO-FEDER Grant MTM2012-31714 and the Catalan Grant 2014SGR504. The author MG has been supported by the DFG Collaborative Research Center TRR 109 "Discretization in Geometry and Dynamics". 
tangencies of multidimensional symplectic maps. However, the Newhouse theorem from [4] does not give answer whether these 1-elliptic periodic orbits are generic 1 This fact is very important for the two-dimensional case where an 1-elliptic periodic orbit is elliptic and the genericity means the KAM-stability. The birth of such generic elliptic periodic orbits under homoclinic bifurcations in symplectic two-dimensional maps was established by Mora and Romero in [7].2 In [7] a one parameter family $f_{\mu}$ of two-dimensional symplectic maps was considered such that (i) $f_{0}$ has a saddle fixed point $O$ with multipliers $\lambda$ and $\lambda^{-1}$, where $0<|\lambda|<1$, and the invariant manifolds of $O$ have a quadratic tangency at the points of a homoclinic orbit $\Gamma_{0}$, and (ii) this tangency splits generally when $\mu$ varies. Then, as is shown in [7], there exists an infinite cascade of intervals $\delta_{k}$, $k=\bar{k}, \bar{k}+1, \ldots$ (where $\bar{k}$ is a sufficiently large integer) of values of $\mu$ such that the intervals $\delta_{k}$ accumulate at $\mu=0$ as $k \rightarrow \infty$ and $f_{\mu}$ has at $\mu \in \delta_{k}$ a single-round elliptic orbit of period $k$. Note that all points of this orbit belong to a small fixed neighborhood of the contour $O \cup \Gamma_{0}$ and any such point is a fixed one for the corresponding first return map $T_{k}$. It was shown in [7] that the map $T_{k}$ can be written in some rescaled coordinates in the form

$$
\bar{x}=y, \bar{y}=M_{k}-x-y^{2}+\nu_{k}(y),
$$

where the new coordinates $(x, y)$ and parameter $M_{k} \sim \lambda^{2 k}\left(\mu-\alpha_{k}\right)$ can take arbitrary finite values as $k$ are large and the the coefficient $\alpha_{k}$ and function $\nu_{k}(y)$ tend to zero as $k \rightarrow \infty$ (the latter, along with their derivatives up to order $(r-2)$ if $\left.f_{\mu} \in C^{r}\right)$. Thus, the map $T_{k}$ is asymptotically close $($ as $k \rightarrow \infty)$ to the conservative Hénon map $\bar{x}=y, \bar{y}=M_{k}-x-y^{2}$ that has an elliptic fixed point when $-1<M_{k}<3$. This point is generic for all such values of $M_{k}$ except for $M_{k}=0$ and $M_{k}=1 / 2$ when the map has a fixed point with multipliers $e^{ \pm i \pi / 2}$ and $e^{ \pm i 2 \pi / 3}$, respectively. This result gives immediately the intervals $\delta_{k}$ with border points corresponding to $M_{k}=3$ and $M_{k}=-1$, respectively.

However, the question on coexistence of single-round periodic orbits of different periods, i.e., whether the intervals $\delta_{k}$ with different numbers intersect, was not considered in [7]. Although, this problem is very important, since its solution is only necessary for construction of the bifurcation diagram. We note also that the Hénon map is degenerate with respect to bifurcations of the fixed point with multipliers $e^{ \pm i \pi / 2}$ (the strong resonance 1:4), and this would be strange if the same is valid for the first return map $T_{k}$. Both these problems were solved in the work [8]. However, it was required a development of new technics, in particular, the construction of finite-smooth analogous of the analytical Birkhoff-Moser normal form for a saddle map [27]. First, it was shown in [8] that the intervals $\delta_{k}$ can intersect indeed and, moreover, they can be even nested. In the latter case the phenomenon of global resonance, discovered by S.Gonchenko and Shilnikov in [13, 14], can be observed when, in particular, the symplectic map $f_{0}$ can have simultaneously infinitely many single-round elliptic periodic orbits of all successive periods $k=\bar{k}, \bar{k}+1$,... Second, conditions of nondegeneracy of the resonance 1:4 in the first return maps were found.

In principle, these results were obtained by the way of more accurate calculations of the small terms $\alpha_{k}$ and $\nu_{k}(y)$ in the rescaled form of $T_{k}$. As result, the map of form (1) was deduced with $M_{k}=-d^{-1} \lambda^{-2 k}\left(\mu-\alpha \lambda^{k}\left(1+O\left(k \lambda^{k}\right)\right)\right)+s_{0}+O\left(k \lambda^{k}\right)$ and $\nu_{k}(y)=s_{1} \lambda^{k} y^{3}+\lambda^{2 k} O\left(y^{4}\right)$, where $d \neq 0, \alpha, s_{0}$ and $s_{1}$ are some coefficients (invariants of a homoclinic structure). Then we can immediately see that if $\mu=0$ and $\alpha=0$ (a codimension two bifurcation case), then $M=s_{0}+O\left(k \lambda^{k}\right)$ and, hence, all maps $T_{k}$ are "the same" - all of them are close to the same map

${ }^{1}$ The birth of 2-elliptic generic periodic orbits was proved in [5, 6 for the case of four-dimensional symplectic maps with homoclinic tangencies to saddle-focus fixed points.

${ }^{2}$ Note that an analogous problem was considered in 9, 10, when studying bifurcations of three-dimensional conservative flows with a homoclinic loop of a saddle-focus equilibrium. 
$\bar{x}=y, \bar{y}=s_{0}-x-y^{2}$. In this case, if $-1<s_{0}<3$, every $T_{k}$ has an elliptic fixed point (with $\varphi$ close to arccos $\left(1-\sqrt{1+s_{0}}\right)$ ). Note that the cubic term $s_{1}$ responses for nondegeneracy of the resonance 1:4 (if $s_{1} \lambda^{k}>0$ the point is a saddle with 8 separatrices, if $s_{1} \lambda^{k}<0$ the point is of elliptic type, i.e. KAM-stable).

In this paper we consider area preserving maps defined on a non-orientable surface $\mathcal{M}_{2}$ and study their homoclinic bifurcations 3 As in the paper [8], we construct bifurcation diagrams for single-round periodic orbits and study the phenomenon of "global resonance". First of all, we establish the theorem on cascade of elliptic periodic orbits, Theorem 1. However, we note that, unlike the symplectic case, the first return maps $T_{k}$ can be nonorientable. We note that, in the case under consideration, the maps $T_{k}$ are rescaled to a map asymptotically close (as $k \rightarrow \infty$ ) to the nonorientable conservative Hénon map $\bar{x}=y, \bar{y}=M+x-y^{2}$ (see Lemma 1). Thus, $T_{k}$ can not have elliptic fixed points. However, $T_{k}^{2}$ can have and, therefore, cascades from Theorem 1 relate to double-round elliptic points. In Theorem 2 we generalize results of Theorem 1 for twoparameter families $f_{\mu, \alpha}$ with governing parameters $\mu$ and $\alpha$ (see formula (11) for $\alpha$ ) and deduce the result, Theorem [3, about the existence of infinitely many double-round elliptic periodic orbits of all successive even periods beginning from some number.

We note also that the problem under consideration, as in [13, 14, 8], is related to the Poincaré conjecture [15] on the density of stable (elliptic for the two-dimensional case) periodic orbits in the phase space of non-integrable Hamiltonian systems. This Poincaré problem is wide open.4 Therefore, the above mentioned phenomenon of global resonance (the coexistence of elliptic periodic orbits of all periods) can be considered as quite relevant. There are few explicit criteria for the existence of infinitely many elliptic periodic orbits, and this gives a rare opportunity for the construction of Hamiltonian systems with a given structure of stable modes. From this point of view, it is important that in the case considered in our paper, the global resonance is organized in a quite different way: we give an explicit criterion for the existence of elliptic periodic orbits of all even periods. It is worth mentioning that related problems on the coexistence of large number of elliptic periodic orbits in Hamiltonian systems are quite popular, see e.g. the paper [20] and the corresponding references in it.

Note also that non-orientable APMs appear naturally when restricting a multidimensional symplectic map onto a two-dimensional non-orientable surface as well as when factorizing orientable symplectic maps by a discrete symmetry group. For example, when a map (not necessarily symplectic one) admits certain symmetries, it can be expressed as some even power of a simpler non-orientable map and, thus, the study of the latter map becomes very important, see e.g. [21, 22].

\footnotetext{
${ }^{3}$ Notice that these maps cannot be symplectic due to the lack of orientation on $\mathcal{M}_{2}$.

${ }^{4}$ In this connection, we note that for (multidimensional) $C^{1}$-smooth symplectic diffeomorphisms given on compact manifolds, the following properties are generic: 1) hyperbolic periodic points are dense in the phase space, [16]; 2) every hyperbolic periodic orbit has a transverse homoclinic point in any neighborhood of any point of the phase space, [17, 18; 3 ) if a symplectic diffeomorphism $f$ is not Anosov, then the 1-elliptic periodic points of $f$ are dense in the phase space, 4. It is worth remarking that the above-mentioned $C^{1}$ generic properties can become nontypical if one requires a greater smoothness. Thus, for example, according to the KAM-theory, elliptic periodic orbits of $C^{r}$-smooth two-dimensional symplectic diffeomorphisms are generically stable at $r \geq 5$ ([19]), whereas, by property 2), all periodic elliptic orbits of generic $C^{1}$-diffeomorphisms are unstable.
} 


\section{Statement of the problem and main results.}

Consider a $C^{r}$-smooth $(r \geq 3)$ APM $f_{0}$ defined on a non-orientable surface $\mathcal{M}_{2}$ and satisfying the following conditions.

A. $f_{0}$ has a saddle fixed point $O$ with multipliers $\lambda$ and $\lambda^{-1}$, where $|\lambda|<1$.

B. $f_{0}$ has a homoclinic orbit $\Gamma_{0}$ where the stable and unstable invariant manifolds of the saddle $O$ have a quadratic tangency.

Condition A means that there exists a neighborhood (disk) $U_{0}$ of the point $O$ in which the map $T_{0}=\left.f_{0}\right|_{U_{0}}$ is symplectic, i.e., area-preserving and orientable. The map $T_{0}$ is called the local map, it is a saddle map that has the point $O$ as a fixed one. By condition $\mathrm{B}$, the stable $W^{s}$ and unstable $W^{u}$ invariant manifolds of $O$ intersect non-transversally at the points of $\Gamma_{0}$. Infinitely many such homoclinic points are inside $U_{0}$. We take a pair of these points: $M^{+} \in W_{\text {loc }}^{s}$ and $M^{-} \in W_{l o c}^{u}$. Then a natural number $n_{0}$ exists such that $M^{+}=f_{0}^{n_{0}}\left(M^{-}\right)$. Let $\Pi^{+} \subset U_{0}$ and $\Pi^{-} \subset U_{0}$ be sufficiently small neighborhoods of the points $M^{+}$and $M^{-}$, respectively. The map $T_{1}=\left.f_{0}^{n_{0}}\right|_{\Pi^{-}}: \Pi^{-} \rightarrow \Pi^{+}$is called the global map. Evidently, the map $T_{1}$ is area-preserving (in the symplectic coordinates on $U_{0}$ ). However, emphasizing the non-orientability of phase space, we assume that

C. The map $T_{0}$ is symplectic in $U_{0}$, whereas the map $T_{1}$ is area-preserving and non-orientable.

Let $\mathcal{H}_{0}$ be a (codimension one) bifurcation manifold composed of area-preserving $C^{r}$-maps on $\mathcal{M}_{2}$ close to $f_{0}$ and such that every map of $\mathcal{H}_{0}$ has a nontransversal homoclinic orbit close to $\Gamma_{0}$. Let $f_{\varepsilon}$ be a family of area-preserving $C^{r}$-maps that contains the map $f_{0}$ at $\varepsilon=0$. We suppose that the family depends smoothly on parameters $\varepsilon=\left(\varepsilon_{1}, \ldots, \varepsilon_{m}\right)$ and satisfies the following condition.

D. The family $f_{\varepsilon}$ is transverse to $\mathcal{H}_{0}$ at $\varepsilon=0$.

Let $U$ be a small neighborhood of $O \cup \Gamma_{0}$ which consists of the small disk $U_{0}$ containing $O$ and a number of small disks surrounding those points of $\Gamma_{0}$ that do not lie in $U_{0}$ (see Figure 1).

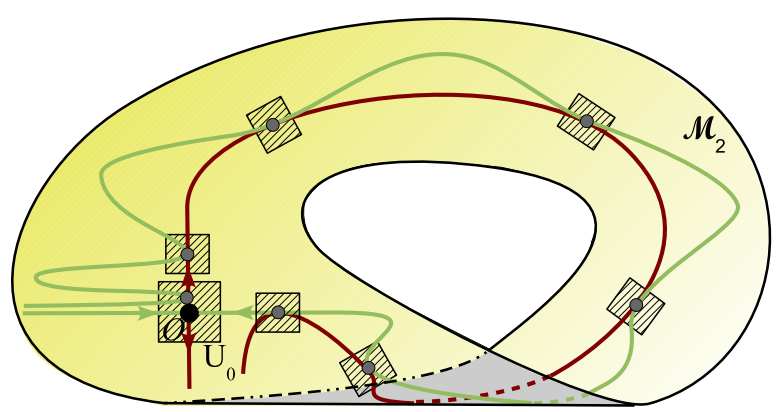

(a)

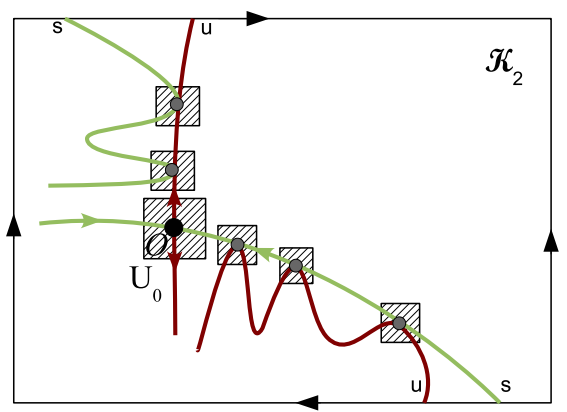

(b)

Figure 1: An example of non-orientable APM (a) on the Möbius band, (b) on the Klein bottle having a quadratic homoclinic tangency at the points of a homoclinic orbit $\Gamma_{0}$. Some of these homoclinic points are shown as grey circles. Also a small neighborhood of the set $O \cup \Gamma_{0}$ is shown as the union of a number of "squares". 
Definition 1. A periodic or homoclinic orbit entirely lying in $U$ is called p-round if it has exactly $p$ intersection points with any disk of the set $U \backslash U_{0}$.

In this paper we study bifurcations of single-round $(p=1)$ periodic orbits in the families $f_{\varepsilon}$. Note that every point of such an orbit can be considered as a fixed point of the corresponding first return map. Such a map is usually constructed as a superposition $T_{k}=T_{1} T_{0}^{k}$ of two maps $T_{0} \equiv T_{0}(\varepsilon)=\left.f_{\varepsilon}\right|_{U_{0}}$ and $T_{1} \equiv T_{1}(\varepsilon)=f_{\varepsilon}^{n_{0}}: \Pi^{-} \rightarrow U_{0}$. Thus, any fixed point of $T_{k}$ is a point of a single-round periodic orbit for $f_{\varepsilon}$ with period $k+n_{0}$. We will study maps $T_{k}$ for all sufficiently large integer $k$.

Condition $\mathrm{D}$ means that one can introduce in $U_{0}$ some canonical coordinates $(x, y)$ such that $J\left(T_{0}\right) \equiv+1$ and $J\left(T_{1}\right) \equiv-1$, where $J(T)$ is the Jacobian of map $T$. Moreover, we can introduce coordinates $(x, y)$ in such a way that the local map $T_{0}$ can be written in one of the so-called finitely smooth normal forms provided by the following lemma.

Lemma 1. 8] For any given integer $n$ (such that $n<r / 2$ or $n$ is arbitrary for $r=\infty$ or $r=\omega-$ the real analytic case), there is a canonical change of coordinates, of class $C^{r}$ for $n=1$ or $C^{r-2 n}$ for $n \geq 2$, that brings $T_{0}$ to the following form

$$
\begin{aligned}
& \bar{x}=\lambda x\left(1+\beta_{1} \cdot x y+\cdots+\beta_{n} \cdot(x y)^{n}\right)+x^{n+1} y^{n} O(|x|+|y|), \\
& \bar{y}=\lambda^{-1} y\left(1+\hat{\beta}_{1} \cdot x y+\cdots+\hat{\beta}_{n} \cdot(x y)^{n}\right)+x^{n} y^{n+1} O(|x|+|y|) .
\end{aligned}
$$

The smoothness of these coordinate changes with respect to parameters can be decreased by 2 unities, i.e., it is $C^{r-2}$ for $n=1$ or $C^{r-2 n-2}$ for $n \geq 2$, respectively.

In these coordinates, the equations of $W_{l o c}^{s} \cap U_{0}$ and $W_{l o c}^{u} \cap U_{0}$ are $y=0$ and $x=0$, respectively. Moreover, the normal forms (2) are very suitable for effective calculation of maps $T_{0}^{k}:\left(x_{0}, y_{0}\right) \rightarrow$ $\left(x_{k}, y_{k}\right)$ with sufficiently large integer $k$. Indeed, the following result is valid.

Lemma 2. 8] Let $T_{0}$ be given by (2), then the map $T_{0}^{k}$ can be written, for any integer $k$, in the so-called cross-form:

$$
\begin{aligned}
& x_{k}=\lambda^{k} x_{0} \cdot R_{n}^{(k)}\left(x_{0} y_{k}, \varepsilon\right)+\lambda^{(n+1) k} P_{n}^{(k)}\left(x_{0}, y_{k}, \varepsilon\right), \\
& y_{0}=\lambda^{k} y_{k} \cdot R_{n}^{(k)}\left(x_{0} y_{k}, \varepsilon\right)+\lambda^{(n+1) k} Q_{n}^{(k)}\left(x_{0}, y_{k}, \varepsilon\right),
\end{aligned}
$$

where

$$
R_{n}^{(k)} \equiv 1+\tilde{\beta}_{1}(k) \lambda^{k} x_{0} y_{k}+\cdots+\tilde{\beta}_{n}(k) \lambda^{n k}\left(x_{0} y_{k}\right)^{n}
$$

$\tilde{\beta}_{i}(k), i=1, \ldots, n$, are some polynomials (of degree $i$ ) with respect to $k$ with coefficients depending on $\beta_{1}, \ldots, \beta_{i}$, e.g. $\tilde{\beta}_{1}=\beta_{1} k, \tilde{\beta}_{2}=\beta_{2} k-\frac{1}{2} \beta_{1}^{2} k^{2}, \ldots$, and the functions $P_{n}^{(k)}, Q_{n}^{(k)}=o\left(x_{0}^{n} y_{k}^{n}\right)$ are uniformly bounded in $k$ along with all their derivatives with respect to coordinates up to order either $(r-2)$ for $n=1$ or $(r-2 n-1)$ for $n \geq 2$.

Remark 3. 1) The normal form of the first order $(n=1)$ for $T_{0}$

$$
\begin{aligned}
& \bar{x}=\lambda x\left(1+\beta_{1} \cdot x y\right)+x^{2} y O(|x|+|y|), \\
& \bar{y}=\lambda^{-1} y\left(1-\beta_{1} \cdot x y\right)+x y^{2} O(|x|+|y|)
\end{aligned}
$$

is well known from [7, 24] where it was proved the existence of normalizing $C^{r-1}$-coordinates. The existence of $C^{r}$-smooth canonical changes of coordinates (which are $C^{r-2}$-smooth with respect to parameters) bringing a symplectic saddle map to form (5) was proved in [25]. 
2) Note that form (2) can be considered as a finitely smooth approximation of the analytical Moser normal form

$$
\bar{x}=\lambda(\varepsilon) x \cdot B(x y, \varepsilon), \quad \bar{y}=\lambda^{-1}(\varepsilon) y \cdot B^{-1}(x y, \varepsilon),
$$

taking place for $\lambda>0$ [27], where $B(x y, \varepsilon)=1+\beta_{1} \cdot x y+\cdots+\beta_{n} \cdot(x y)^{n}+\cdots$. Since the form (6) is integrable (e.g. it has integral $x y$ ), one can easily write the corresponding formula (5) for this case, see [11].

In the coordinates of Lemma 1, we can write $M^{+}=\left(x^{+}, 0\right), M^{-}=\left(0, y^{-}\right)$. Without loss of generality, we assume that $x^{+}>0$ and $y^{-}>0$. Let the neighborhoods $\Pi^{+}$and $\Pi^{-}$of the homoclinic points $M^{+}$and $M^{-}$, respectively, be sufficiently small such that $T_{0}\left(\Pi^{+}\right) \cap \Pi^{+}=\emptyset$, $T_{0}^{-1}\left(\Pi^{-}\right) \cap \Pi^{-}=\emptyset$. Then, as usual (see e.g. [1, [26]), the map from $\Pi^{+}$into $\Pi^{-}$by orbits of $T_{0}$ is defined, for all sufficiently small $\varepsilon$, on the set consisting of infinitely many strips $\sigma_{k}^{0} \equiv \Pi^{+} \cap T_{0}^{-k} \Pi^{-}$, $k=\bar{k}, \bar{k}+1, \ldots$ The image of $\sigma_{k}^{0}$ under $T_{0}^{k}$ is the strip $\sigma_{k}^{1}=T_{0}^{k}\left(\sigma_{k}^{0}\right) \equiv \Pi^{-} \cap T_{0}^{k} \Pi^{+}$. As $k \rightarrow \infty$, the strips $\sigma_{k}^{0}$ and $\sigma_{k}^{1}$ accumulate on $W_{l o c}^{s}$ and $W_{l o c}^{u}$, respectively.

We can write the global map $T_{1}(\varepsilon): \Pi^{-} \rightarrow \Pi^{+}$as follows (in the coordinates of Lemma 1)

$$
\bar{x}-x^{+}=F\left(x, y-y^{-}, \varepsilon\right), \quad \bar{y}=G\left(x, y-y^{-}, \varepsilon\right),
$$

where $F(0)=0, G(0)=0$. Besides, we have that $G_{y}(0)=0, G_{y y}(0)=2 d \neq 0$ which follows from the fact (condition B) that at $\varepsilon=0$ the curve $T_{1}\left(W_{l o c}^{u}\right):\left\{\bar{x}-x^{+}=F\left(0, y-y^{-}, 0\right), \bar{y}=\right.$ $\left.G\left(0, y-y^{-}, 0\right)\right\}$ has a quadratic tangency with $W_{l o c}^{s}:\{\bar{y}=0\}$ at $M^{+}$. When the parameters $\varepsilon$ vary this tangency can split and, moreover, by condition $C$, we can introduce the corresponding splitting parameter as $\mu \equiv G(0,0, \varepsilon)$. Accordingly, we can write

$$
\begin{aligned}
F\left(x, y-y^{-}, \varepsilon\right)= & a x+b\left(y-y^{-}\right)+e_{20} x^{2}+e_{11} x\left(y-y^{-}\right)+e_{02}\left(y-y^{-}\right)^{2}+\text { h.o.t. } \\
G\left(x, y-y^{-}, \varepsilon\right)= & \mu+c x+d\left(y-y^{-}\right)^{2}+f_{20} x^{2}+f_{11} x\left(y-y^{-}\right)+f_{30} x^{3} \\
& +f_{21} x^{2}\left(y-y^{-}\right)+f_{12} x\left(y-y^{-}\right)^{2}+f_{03}\left(y-y^{-}\right)^{3}+\text { h.o.t. }
\end{aligned}
$$

where the coefficients $a, b, \ldots, f_{03}$ (as well as $x^{+}$and $y^{-}$) depend smoothly on $\varepsilon$. Note also that

$$
J\left(T_{1}\right)=\operatorname{det}\left(\begin{array}{cc}
F_{x} & F_{y} \\
G_{x} & G_{y}
\end{array}\right) \equiv-1
$$

since $T_{1}$ is non-orientable map by condition $\mathrm{C}$. In particular, we have

$$
\begin{gathered}
b c \equiv+1, \\
\tilde{R}=\left(2 a+2 e_{02} / b d-b f_{11} / d\right) \equiv 0
\end{gathered}
$$

It is clear from (17) and (8) that $\mu$ is the parameter of splitting of manifolds $W^{s}\left(O_{\varepsilon}\right)$ and $W^{u}\left(O_{\varepsilon}\right)$ with respect to the homoclinic point $M^{+}$. Indeed, the curve $l_{u}=T_{1}\left(W_{l o c}^{u} \cap \Pi^{-}\right)$has the equation $l_{u}: \bar{y}=\mu+\frac{d}{b^{2}}\left(\bar{x}-x^{+}\right)^{2}\left(1+O\left(\bar{x}-x^{+}\right)\right)$. Since the equation of $W_{l o c}^{s}$ is $y=0$ for all (small) $\varepsilon$, it implies that the manifolds $T_{1}\left(W_{l o c}^{u}\right)$ and $W_{l o c}^{s}$ do not intersect for $\mu d>0$, intersect transversally at two points for $\mu d<0$, and have a quadratic tangency (at $M^{+}$) for $\mu=0$. Besides, since the strips $\sigma_{k}^{1}$ accumulate on the segment $W_{l o c}^{u} \cap \Pi^{-}$as $k \rightarrow \infty$, it follows that the images $T_{1}\left(\sigma_{k}^{1}\right)$ of $\sigma_{k}^{1}$ under $T_{1}$ have a horseshoe form and, moreover, horseshoes $T_{1}\left(\sigma_{k}^{1}\right)$ accumulate on $l_{u}$ as $k \rightarrow \infty$. Therefore, the first return maps $T_{k}=T_{1} T_{0}^{k}: \sigma_{k}^{0} \rightarrow \sigma_{k}^{0}$ are, in fact, conservative horseshoe maps with the Jacobian -1. Geometrically, the action of this map looks as in Figure 2 ,

When $\mu$ varies near zero infinitely many bifurcations of horseshoes creation (destruction) occur. In this paper we study these bifurcations and show that they include birth (disappearance) of elliptic periodic points. 

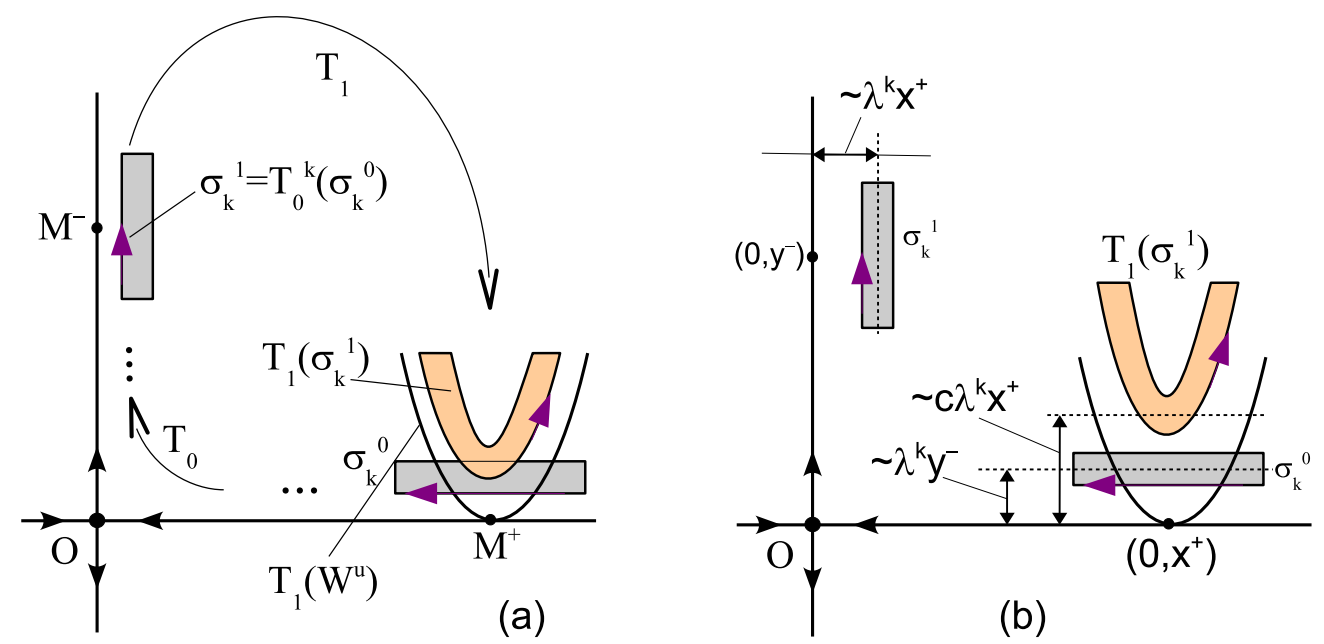

Figure 2: (a) Actions of the local and global maps $T_{0}$ and $T_{1}$ in $U_{0}$. Under the map $T_{0}^{k}$ some strip $\sigma_{k}^{0}$ near $M^{+}$is transformed into a strip $\sigma_{k}^{1}$ lying near $M^{-}$. Under the global map $T_{1}$ the strip $\sigma_{k}^{1}$ is transformed into a horseshoe $T_{1}\left(\sigma_{1}\right)$. The latter map is non-orientable, i.e., changes the orientation of the boundary of the horseshoe with respect to the orientation of the pre-image $\sigma_{k}^{1}$. The direction of the orientation is indicated by the arrows. We assume that $T_{0}$ is orientable, therefore, the boundary orientation of $\sigma_{k}^{0}$ and $\sigma_{k}^{1}$ is the same. (b) To a reciprocal position of the strips $\sigma_{k}^{0}$ and horseshoes $T_{1}\left(\sigma_{k}^{1}\right)$. The strips $\sigma_{k}^{0}$ and $\sigma_{k}^{1}$ are posed on a distance $\lambda^{k} y^{-}(1+\ldots)$ from $W_{l o c}^{s}$ and $\lambda^{k} x^{+}(1+\ldots)$ from $W_{l o c}^{u}$, respectively. Hence, the top of horseshoe $T_{1}\left(\sigma_{k}^{1}\right)$ are posed on a distance $c \lambda^{k} x^{+}(1+\ldots)$ from $W_{l o c}^{s}$.

However, we can also see that these horseshoe bifurcations must have different scenarios depending on the type of the initial homoclinic tangency. Indeed, at $\mu=0$ the character of the reciprocal position of the strips $\sigma_{k}^{0}$ and their horseshoes $T_{1}\left(\sigma_{k}^{1}\right)$ is essentially defined by the signs of the parameters $\lambda, c$ and $d$. Moreover, by this feature, we can select 6 different cases of APMs with quadratic homoclinic tangencies. The corresponding examples are shown in Figures 3 and 4. Note that in the cases with $\lambda<0$ we can always consider $d$ to be positive: if $d$ is negative for the given pair of homoclinic points, $M^{+}$and $M^{-}$, we can take another pair of points, like $\left\{T_{0}\left(M^{+}\right), M^{-}\right\}$or $\left\{M^{+}, T_{0}^{-1}\left(M^{-}\right)\right\}$, for which the corresponding $d^{\prime}$ becomes positive.

Note that in the cases with $c<0$, see Figure 3, the reciprocal position of all the strips $\sigma_{j}^{0}$ and their horseshoes $T_{1}\left(\sigma_{j}^{1}\right)$ at $\mu=0$ is defined quite simply: $\sigma_{j}^{0} \cap T_{1}\left(\sigma_{j}^{1}\right)=\emptyset$ if $\lambda>0, d<0$; the strips $\sigma_{j}^{0}$ and horseshoes $T_{1}\left(\sigma_{j}^{1}\right)$ have regular intersections if $\lambda>0, d>0$; the corresponding intersections are either regular for even $j$ or empty for odd $j$ if $\lambda<0, d>0$. Recall that regular intersection means here (by [28] and [29]) that the set $\sigma_{j}^{0} \cap T_{1}\left(\sigma_{j}^{1}\right)$ consists of two connected components and, moreover, the first return map $T_{j} \equiv T_{1} T_{0}^{j}: \sigma_{j}^{0} \mapsto \sigma_{j}^{0}$ is the Smale horseshoe map: its nonwandering set $\Omega_{j}$ is hyperbolic and $\left.T_{j}\right|_{\Omega_{j}}$ is topologically conjugate to the Bernoulli shift with two symbols (for more details see [29, 8]). Therefore, we can say that every map $f_{0}$ in the case $c<0, d>0$ has infinitely many horseshoes $\Omega_{j}$, where $j$ runs for all sufficiently large positive integers (respectively, even positive integers) in the case $\lambda>0$ (respectively, in the case $\lambda<0$ ). On the other hand, every map $f_{0}$ with $\lambda>0, c<0, d<0$ has no horseshoes at all (in a small neighborhood $U$ ).

In the cases of homoclinic tangencies with $c>0$, see Figure 4 , the reciprocal position of the strips $\sigma_{j}^{0}$ and horseshoes $T_{1}\left(\sigma_{j}^{1}\right)$ depends also on other invariant quantities of the homoclinic 


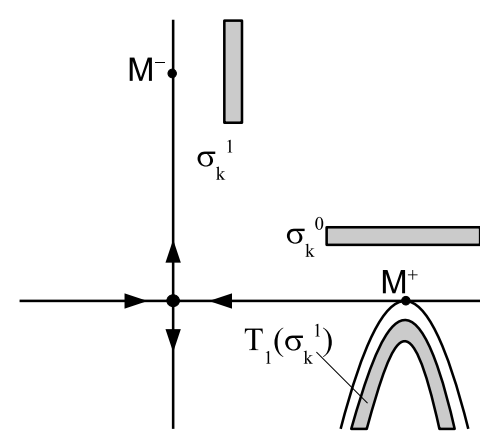

(a) $\lambda>0, c<0, d<0$

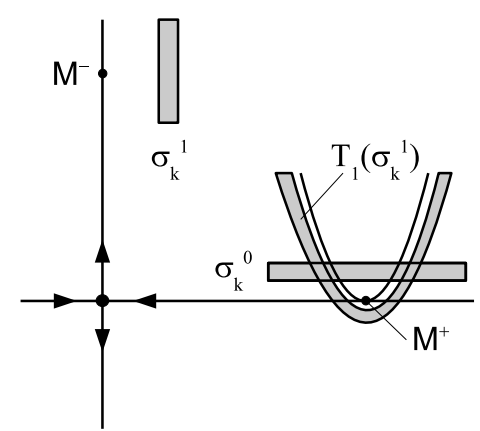

(b) $\lambda>0, c<0, d>0$

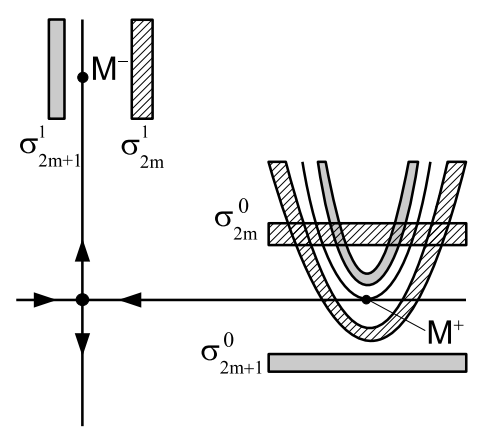

(c) $\lambda<0, c<0, d>0$

Figure 3: Types of APMs with a homoclinic tangency for $c<0$.

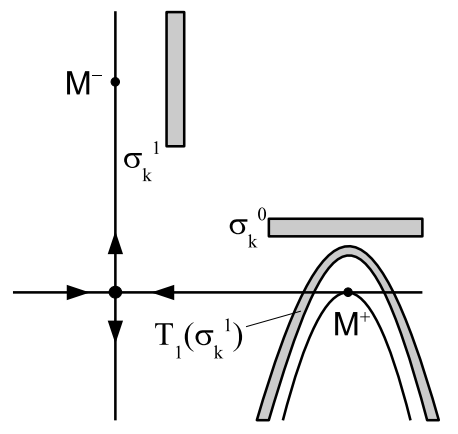

(a) $\lambda>0, c>0, d<0$

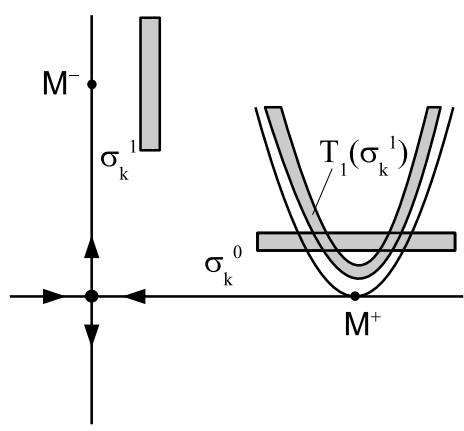

(b) $\lambda>0, c>0, d>0$

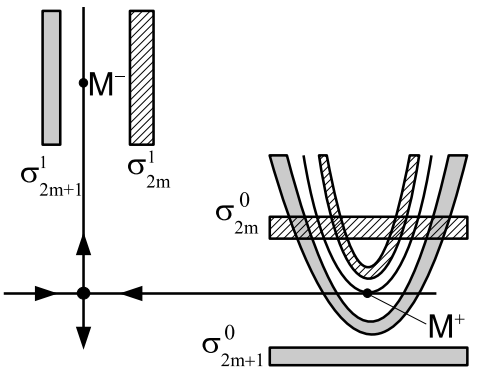

(c) $\lambda<0, c>0, d>0$

Figure 4: Types of APMs with a homoclinic tangency for $c>0$.

structure, the most important being, [13, 14, 28,

$$
\alpha=\frac{c x^{+}}{y^{-}}-1 \text {. }
$$

First of all, notice that the sign of $\alpha$ is very important. For example, in Figure 5 it is shown the reciprocal position of the strips $\sigma_{j}^{0}$ and horseshoes $T_{1}\left(\sigma_{j}^{1}\right)$ (with sufficiently large $j$ ) for various values of $\alpha$ for the case $\lambda>0, c>0, d>0$. Thus, we can see that 5

- if $\alpha<0$, then $f_{0}$ has infinitely many horseshoes $\Omega_{j}$;

- if $\alpha>0$, then there exists a neighborhood $U\left(O \cap \Gamma_{0}\right)$ in which the dynamics of $f_{0}$ is trivial: only orbits $O$ and $\Gamma_{0}$ do not leave $U$ under iterations of $f_{0}$.

Thus, $\alpha=0$ is a bifurcation value, since infinitely many horseshoes appear (disappear) when varying $\alpha$ near zero (even without splitting the initial tangency).

\footnotetext{
${ }^{5}$ These results can be easily explained by using the geometry of Figure 2 Thus, if $c \lambda^{k} x^{+}>\lambda^{k} y^{-}$, i.e., $\alpha>0$, the top of the horseshoe is above the strip $\sigma_{k}^{0}$. This means that $f_{0}$ has no horseshoes and the dynamics is trivial. However, if $c \lambda^{k} x^{+}<\lambda^{k} y^{-}$, i.e., $\alpha<0$, the top of the horseshoe is below the strip $\sigma_{k}^{0}$. Geometrically, it means that $f_{0}$ has infinitely many horseshoes (for every sufficiently large $k$ ). A rigorous proof requires quite elaborate analytical considerations which are not presented here, see e.g. [8, 11, 12, 14].
} 


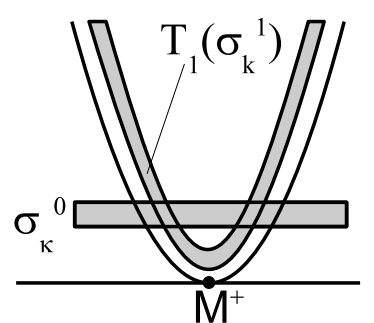

(a) $\alpha<0$

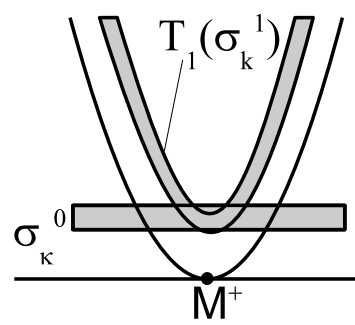

(b) $\alpha=0$

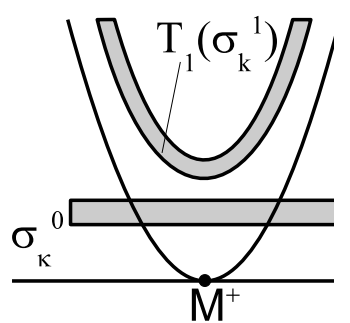

(c) $\alpha>0$

Figure 5: A horseshoe geometry of symplectic maps with a homoclinic tangency in the case $\lambda>0, c>0, d>0$ for various $\alpha$.

Thus, we can draw the following conclusions:

1) the cases of homoclinic tangencies with $c<0$ or with $\alpha \neq 0$ at $c>0$ are "ordinary" and it is sufficient to study bifurcations of single-round periodic orbits only within the framework of one parameter general families (with the parameter $\mu$ );

2) the cases of homoclinic tangencies with $\alpha=0$ at $c>0$ are "special" and it is necessary to consider at least two parameter general unfoldings (for example, with parameters $\mu$ and $\alpha$ ).

In this paper we adhere to this approach and present the following three theorems as our main results in the case under consideration (APMs on non-orientable surfaces).

Theorem 1. Let $f_{0}$ be an APM satisfying conditions $A, B$ and $D$ and $f_{\mu}$ be a one parameter family of APMs that unfolds generally (under condition $C$ ) at $\mu=0$ the quadratic homoclinic tangency. Then for any interval $\left(-\mu_{0}, \mu_{0}\right)$ of values of $\mu$, there exists a positive integer $\bar{k}$ such that the following holds:

1. All APMs close to $f_{0}$ have no single-round elliptic periodic orbits, while there exist intervals $\mathrm{e}_{k}^{2} \subset I_{\varepsilon}, k=\bar{k}, \bar{k}+1, \ldots$, such that the map $f_{\mu}$ has at $\mu \in \mathrm{e}_{k}^{2}$ a double-round elliptic periodic orbit, of period $2\left(k+n_{0}\right)$, which corresponds to a 2-periodic point of the first return map $T_{k}$.

2. The intervals $\mathrm{e}_{k}^{2}$ accumulate at $\mu=0$ as $k \rightarrow \infty$ and do not intersect for sufficiently large and different integer $k$ if $c<0$, or $\alpha \neq 0$ in the case $c>0$.

3. Any interval $\mathrm{e}_{k}^{2}$ has border points $\mu=\mu_{k}^{2+}$ and $\mu=\mu_{k}^{2-}$ where the map $f_{\mu}$ has a singleround periodic orbit (of period $\left(k+n_{0}\right)$ ) with multipliers +1 and -1 at $\mu=\mu_{k}^{2+}$ and a double-round periodic orbit (of period $2\left(k+n_{0}\right)$ ) with double multiplier -1 at $\mu=\mu_{k}^{2-}$. See Figure 6 .

4. The angular argument $\varphi$ of the multipliers $e^{ \pm i \varphi}$ of the elliptic periodic orbits for $\mu \in \mathrm{e}_{k}^{2}$ depends monotonically on $\mu$ and the elliptic orbit is generic (KAM-stable) for all such $\mu$, except for those $\varphi=\varphi(\mu)$ such that $\varphi(\mu)=\frac{\pi}{2}, \frac{2 \pi}{3}$, $\arccos \left(-\frac{1}{4}\right)$.

Note that Theorem 1 does not give answer to the question of the mutual position of the intervals $\mathrm{e}_{k}^{2}$ in the critical case $\alpha=0$. But this value of $\alpha$ is quite important, since it is related to the coexistence of elliptic orbits of different periods. Therefore, we assume now that $f_{0}$ is a map satisfying conditions $\mathrm{A}, \mathrm{B}$ and $\mathrm{D}$ with $\alpha=0$ and consider a two parameter family $\left\{f_{\mu, \alpha}\right\}$ which is a general unfolding for the initial tangency with $\alpha=0$. Let $D_{\epsilon}$ be a sufficiently small neighborhood (of diameter $\epsilon>0$ ) of the origin in the parameter plane $(\mu, \alpha)$. Then the following result holds.

Theorem 2. In $D_{\epsilon}$, for any $\epsilon>0$, there exist infinitely many open domains $E_{k}^{2}$ (strips) such that if $(\mu, \alpha) \in E_{k}^{2}$, then the map $f_{\mu, \alpha}$ has a double-round elliptic orbit of period $2\left(k+n_{0}\right)$ (corresponding 


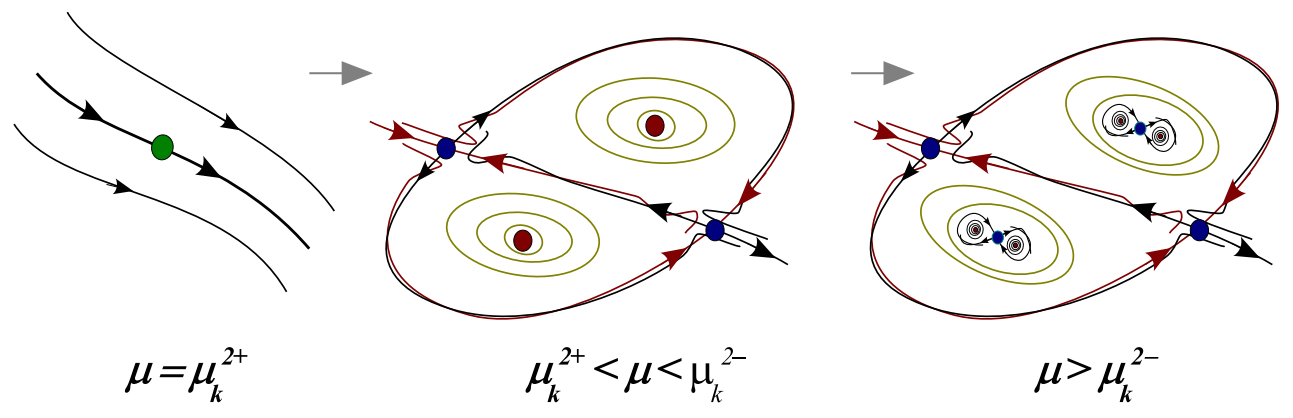

Figure 6: Bifurcation scenarios in the first return maps $T_{k}$ according to item 3 of Theorem 1, We show here that the birth of the elliptic 2-periodic point takes place when increasing $\mu$, while for some types of homoclinic tangencies it can occur at decreasing $\mu$. Since $T_{k}$ is a non-orientable map, then the value $\mu=\mu_{k}^{2+}$ corresponds to the appearance of a fixed point with multipliers +1 and -1 . This point bifurcates into four points, two saddle fixed ones and other two points forming an elliptic 2-periodic orbit, when $\mu \in \mathrm{e}_{k}^{2}$. The value $\mu=\mu_{k}^{2-}$ corresponds to the period doubling bifurcation of this elliptic 2-periodic orbit.

to a elliptic 2-periodic orbit of the first return map $T_{k}$ ). The domains $E_{k}^{2}$ accumulate at the axis $\mu=0$ as $k \rightarrow \infty$, all of them are mutually crossed and intersect the axis $\mu=0$. Every domain $E_{k}^{2}$ has two smooth boundaries, the bifurcation curves $L_{k}^{2+}$ and $L_{k}^{2-}$, which correspond, respectively, to the existence of a single-round nondegenerate periodic orbit with double multipliers +1 and a double-round nondegenerate periodic orbit with double multipliers -1 .

In Figure 7 some qualitative illustrations to Theorem 2 are shown for the cases where (a) $\lambda>0, c>0, d>0$ and (b) $\lambda<0, c>0, d>0$.

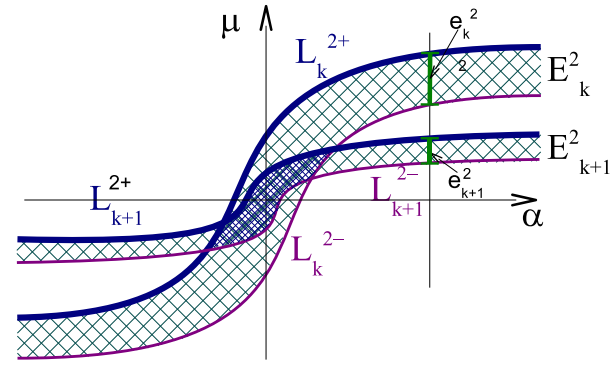

(a) $\lambda=\gamma^{-1}>0, c>0, d>0$

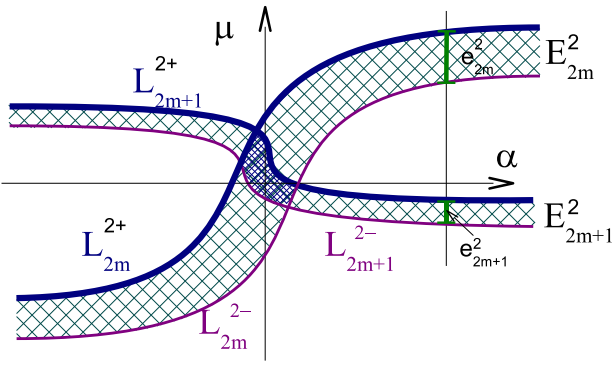

(b) $\lambda=\gamma^{-1}<0, c>0, d>0$

Figure 7: Elements of the bifurcation diagrams for the families $f_{\mu, \alpha}$.

We introduce now the following quantity

$$
s_{0}=d x^{+}\left(a c+f_{20} x^{+}\right)-\frac{1}{4}\left(f_{11} x^{+}\right)^{2},
$$

which is calculated through the coefficients of the global map $T_{1}$, see formula (88), and plays an important role in the global dynamics of the map $f_{0}$ with $\alpha=0$. 
Theorem 2 shows that elliptic (double-round) periodic orbits of different periods can coexist when values of $\mu$ and $\alpha$ vary near zero. Moreover, infinitely many such orbits can coexist, in principle, at the global resonance $\mu=0, \alpha=0$. The following theorem give us sufficient conditions for this phenomenon.

Theorem 3. Let $f_{0}$ be an APM satisfying conditions $A, B$ and $D$. We assume also that the resonant condition $\alpha=0$ takes place for $f_{0}$. Then, if $s_{0}$ (see formula (12)) is such that $-1<s_{0}<0$, then there exists a positive integer $\bar{k}$ such that $f_{0}$ has infinitely many double-round elliptic periodic orbits of all successive even periods $2\left(k+n_{0}\right)$, where $k \geq \bar{k}$. Moreover, if $s_{0} \neq-\frac{1}{2} ;-\frac{1}{\sqrt{2}} ;-\frac{5}{8}$, all these elliptic periodic orbits are generic (KAM-stable).

In the rest part of the paper we prove these and related results.

\section{Rescaling Lemma.}

In principle, for the study of bifurcations in the first return map $T_{k}=T_{1} T_{0}^{k}$ we could write it in the initial coordinates using formulas (8) and (3) for the maps $T_{1}$ and $T_{0}^{k}$, respectively, and, after, work with the obtained formulas. However, there is a more effective way for studying bifurcations. Namely, we can bring maps $T_{k}$ to some unified form for all large $k$ using the so-called rescaling method as it has been done in many papers 6 After this, we can study (one time) bifurcations in the unified map and "project" the obtained results onto the first return maps $T_{k}$ for various $k$. This "universal" map is deduced in the following lemma.

Lemma 1. Let $f_{\varepsilon}$ be the family under consideration satisfying conditions $A-D$. Then, for every sufficiently large $k$, the first return map $T_{k}: \sigma_{k}^{0} \rightarrow \sigma_{k}^{0}$ can be brought, by a linear transformation of coordinates and parameters, to the following form

$$
\begin{aligned}
& \bar{X}=Y, \\
& \bar{Y}=M+X-Y^{2}+\frac{f_{03}}{d^{2}} \lambda^{k} Y^{3}+k \lambda^{2 k} \varepsilon_{k}(Y, M) .
\end{aligned}
$$

Map (13) is defined on some asymptotically large domain covering in the limit $k \rightarrow+\infty$ all finite values of $X, Y$ and $M$, the function $\varepsilon_{k}(Y, M)$ is uniformly bounded in $k$ jointly with all their derivatives up to order $(r-4))$, and the following formula takes place for $M$ :

$$
M=-d\left(1+\nu_{k}^{1}\right) \lambda^{-2 k}\left(\mu+\lambda^{k}\left(c x^{+}-y^{-}\right)\left(1+k \beta_{1} \lambda^{k} x^{+} y^{-}\right)\right)-s_{0}+\nu_{k}^{2}
$$

with the coefficient $s_{0}$ satisfying (12) and where $\nu_{k}^{1}=O\left(\lambda^{k}\right), \nu_{k}^{2}=O\left(k \lambda^{k}\right)$ are some asymptotically small coefficients.

Proof. We will use the representation of the symplectic map $T_{0}$ in the "second normal form", i.e., in form (2) for $n=27$ Then the map $T_{0}^{k}: \sigma_{k}^{0} \rightarrow \sigma_{k}^{1}$, for all sufficiently large $k$, can be written in the following form

$$
x_{k}=\lambda^{k} x_{0}\left(1+\beta_{1} k \lambda^{k} x_{0} y_{k}\right)+O\left(k^{2} \lambda^{3 k}\right), \quad y_{0}=\lambda^{k} y_{k}\left(1+\beta_{1} k \lambda^{k} x_{0} y_{k}\right)+O\left(k^{2} \lambda^{3 k}\right) .
$$

\footnotetext{
${ }^{6}$ see e.g. the papers [10, 17, 11, 15, 30, 12, 14, where the rescaling method was applied for the conservative case.

${ }^{7}$ Clearly, we lose a little in a smoothness, since the second order normal form is $C^{r-2}$ only, see Lemma 1 . However, we get more important information on form of the first return maps. On the other hand, our considerations cover also the $C^{\infty}$ and real analytical cases.
} 
Then, using formulae (8) and (15), we can write the first return map $T_{k}: \sigma_{k}^{0} \rightarrow \sigma_{k}^{0}$ in the following form

$$
\begin{aligned}
& \bar{x}-x^{+}= a \lambda^{k} x+b\left(y-y^{-}\right)+e_{02}\left(y-y^{-}\right)^{2}+ \\
&+O\left(k|\lambda|^{2 k}|x|+\left|y-y^{-}\right|^{3}+|\lambda|^{k}|x|\left|y-y^{-}\right|\right), \\
& \lambda^{k} \bar{y}(1+\left.k \lambda^{k} \beta_{1} \bar{x} \bar{y}\right)+k \lambda^{3 k} O(|\bar{x}|+|\bar{y}|)= \\
&=\mu+c \lambda^{k} x\left(1+k \lambda^{k} \beta_{1} x y\right)+d\left(y-y^{-}\right)^{2}+\lambda^{2 k} f_{02} x^{2}+ \\
&+\lambda^{k} f_{11}\left(1+k \lambda^{k} \beta_{1} x y\right) x\left(y-y^{-}\right)+\lambda^{k} f_{12} x\left(y-y^{-}\right)^{2}+f_{03}\left(y-y^{-}\right)^{3}+ \\
&+ \\
&+O\left(\left(y-y^{-}\right)^{4}+\lambda^{2 k}|x|\left|y-y^{-}\right|+k|\lambda|^{3 k}|x|+k \lambda^{2 k}|x|\left|y-y^{-}\right|^{2}\right),
\end{aligned}
$$

where $x=x_{0}, y=y_{k}$.

Below, we will denote by $\alpha_{k}^{i}, i=1,2, \ldots$, some coefficients asymptotically small in $k$ such that $\alpha_{k}^{i}=O\left(k \lambda^{k}\right)$. Now we shift the coordinates

$$
\eta=y-y^{-}, \xi=x-x^{+}-\lambda^{k} x^{+}\left(a+\alpha_{k}^{1}\right),
$$

in order to cancel the constant term (independent of coordinates) in the first equation of (16). Thus, (16) is recast as follows

$$
\begin{gathered}
\bar{\xi}=a \lambda^{k} \xi+b \eta+e_{02} \eta^{2}+O\left(k \lambda^{2 k}|\xi|+|\eta|^{3}+|\lambda|^{k} O(|\xi||\eta|)\right) \\
\lambda^{k} \bar{\eta}\left(1+\alpha_{k}^{2}\right)+k \lambda^{2 k} O\left(|\bar{\xi}|+\bar{\eta}^{2}\right)+k \lambda^{3 k} O(|\bar{\eta}|)= \\
=M_{1}+c \lambda^{k} \xi\left(1+\alpha_{k}^{3}\right)+\eta^{2}\left(d+\lambda^{k} f_{12} x^{+}\right)+\lambda^{k} \eta\left(f_{11} x^{+}+\alpha_{k}^{4}\right)+\lambda^{k} f_{11} \xi \eta+f_{03} \eta^{3}+ \\
+O\left(\eta^{4}+k|\lambda|^{3 k}|\xi|+k \lambda^{2 k}\left(\xi^{2}+\eta^{2}\right)+\lambda^{k}|\xi| \eta^{2}\right)
\end{gathered}
$$

where

$$
M_{1}=\mu+\lambda^{k}\left(c x^{+}-y^{-}\right)\left(1+k \lambda^{k} \beta_{1} x^{+} y^{-}\right)+\lambda^{2 k} x^{+}\left(a c+f_{02} x^{+}\right)+O\left(k \lambda^{3 k}\right) .
$$

Now, we rescale the variables:

$$
\xi=-\frac{b\left(1+\alpha_{k}^{2}\right)}{d+\lambda^{k} f_{12} x^{+}} \lambda^{k} u, \eta=-\frac{1+\alpha_{k}^{2}}{d+\lambda^{k} f_{12} x^{+}} \lambda^{k} v .
$$

System (17) in coordinates $(u, v)$ is rewritten in the following form

$$
\begin{aligned}
\bar{u}=v+ & a \lambda^{k} u-\frac{e_{02}}{b d} \lambda^{k} v^{2}+O\left(k \lambda^{2 k}\right), \\
\bar{v}=M_{2} & +u\left(1+\alpha_{k}^{5}\right)-v^{2}+ \\
& +v\left(f_{11} x^{+}+\alpha_{k}^{6}\right)-\frac{f_{11} b}{d} \lambda^{k} u v+\frac{f_{03}}{d^{2}} \lambda^{k} v^{3}+O\left(k \lambda^{2 k}\right),
\end{aligned}
$$

where

$$
M_{2}=-\frac{d+\lambda^{k} f_{12} x^{+}}{1+\alpha_{k}^{2}} \lambda^{-2 k} M_{1} .
$$

The following shift of coordinates (we remove the terms linear in $v$ from the second equation)

$$
u_{\text {new }}=u-\frac{1}{2}\left(f_{11} x^{+}+\alpha_{k}^{6}\right), v_{n e w}=v-\frac{1}{2}\left(f_{11} x^{+}+\alpha_{k}^{6}\right),
$$


brings map (20) to the following form

$$
\begin{aligned}
& \bar{u}=v+a \lambda^{k} u-\frac{e_{02}}{b d} \lambda^{k} v^{2}+O\left(k \lambda^{2 k}\right), \\
& \bar{v}=M_{3}+u-v^{2}-\frac{f_{11} b}{d} \lambda^{k} u v+\frac{f_{03}}{d^{2}} \lambda^{k} v^{3}+O\left(k \lambda^{2 k}\right),
\end{aligned}
$$

where

$$
M_{3}=M_{2}+\frac{\left(f_{11} x^{+}\right)^{2}}{4}
$$

Now, we make the following linear change of coordinates

$$
x=u+\tilde{\nu}_{k}^{1} v, y=v-\tilde{\nu}_{k}^{2} u
$$

where

$$
\tilde{\nu}_{k}^{1}=-\frac{e_{02}}{b d} \lambda^{k}, \tilde{\nu}_{k}^{2}=-\frac{e_{02}}{b d} \lambda^{k}-a \lambda^{k}
$$

Then, system (21) is rewritten as

$$
\begin{aligned}
& \bar{x}=y+M_{3} \tilde{\nu}_{k}^{1}+O\left(k \lambda^{2 k}\right), \\
& \bar{y}=M_{3}+x-y^{2}+a \lambda^{k} y-\tilde{R} \lambda^{k} x y+\frac{f_{03}}{d^{2}} \lambda^{k} y^{3}+O\left(k \lambda^{2 k}\right),
\end{aligned}
$$

where $\tilde{R}=\left(2 a+2 e_{02} / b d-b f_{11} / d\right) \equiv 0$ by (10). Hence, map (24) has the following form

$$
\begin{aligned}
& \bar{x}=y+M_{3} \tilde{\nu}_{k}^{1}+O\left(k \lambda^{2 k}\right), \\
& \bar{y}=M_{3}+x-y^{2}+a \lambda^{k} y+\frac{f_{03}}{d^{2}} \lambda^{k} y^{3}+O\left(k \lambda^{2 k}\right),
\end{aligned}
$$

Finally, we make one more shift of coordinates

$$
X=x-\frac{1}{2} a \lambda^{k}-\tilde{\nu}_{k}^{1} M_{3}, \quad Y=y-\frac{1}{2} a \lambda^{k},
$$

in order to cancel in (25) the constant term in the first equation and the term linear in $y$ in the second equation. After this, we obtain the final form (13) of map $T_{k}$ in the rescaled coordinates where formula (14) takes place for the parameter $M$.

\section{Proofs of the main results.}

The bifurcations in the first return maps $T_{k}$ can be studied now by using their normal forms deduced from the rescaling Lemma 1. Since these normal forms coincide up to asymptotically small terms as $k \rightarrow \infty$ with the non-orientable conservative Hénon map, we recall in the next section some necessary results on bifurcations of fixed points in one parameter families of conservative Hénon map in the non-orientable case. 


\subsection{On bifurcations of fixed points in the conservative non-orientable Hénon maps.}

The rescaling Lemma 1 shows that the unified limit form for the first return maps $T_{k}$ is the non-orientable and conservative Hénon map

$$
\bar{x}=y, \bar{y}=M+x-y^{2},
$$

with Jacobian $J=-1$. Bifurcations of fixed points in the conservative Hénon family are well known.

Since the Hénon map (26) is not orientable, it cannot have elliptic fixed points. However, there exist elliptic 2-periodic orbits for $M \in(0,1)$. The map has no fixed points for $M<0$, it has one fixed point $\bar{O}(0,0)$ with multipliers $\nu_{1}=+1, \nu_{2}=-1$ at $M=0$ and two saddle fixed points $\left(\bar{O}_{1}(-\sqrt{M},-\sqrt{M})\right.$ and $\left.\bar{O}_{2}(\sqrt{M}, \sqrt{M})\right)$ at $M>0$. Besides, an elliptic 2-periodic orbit exists for $0<M<1$, consisting of the two points $p_{1}=(-\sqrt{M}, \sqrt{M})$ and $p_{2}=(\sqrt{M},-\sqrt{M})$; the value $M=1$ corresponds to a period doubling bifurcation of this orbit. Note that the elliptic 2-periodic orbit is generic for all $M \in(0,1)$ except for $M=\frac{1}{2}$ and $M=\frac{3}{4}$ which correspond to the strong resonances $1: 4$ and $1: 3$, respectively, and $M=\frac{5}{8}$ which corresponds to the cancellation of the first Birkhoff coefficient at the cycle $\left\{p_{1}, p_{2}\right\}$, see [23].

It is also known (see, e.g., [31, 32]) that if $M>5+2 \sqrt{5}$ (this is only a sufficient condition), then the nonwandering set of map (26) is a Smale horseshoe which is non-orientable for this case.

\subsection{Proof of Theorem 1 .}

The proof is deduced from the rescaling lemma 1. Indeed, since the bifurcations of fixed points of the Hénon map (26) are known, we can use this information directly to recover the bifurcations of the single-round periodic orbits in the family $f_{\mu}$. We only need to know the relations between the parameters of the rescaled map (13) and the initial parameters (i.e., in fact, between $M$ and $\mu)$.

In the case under consideration, the relations between $M$ and $\mu$ are given by formula (14) from which we find $\mu$ as follows

$$
\mu=-\lambda^{k} y^{-} \alpha\left(1+k \beta_{1} \lambda^{k} x^{+} y^{-}\right)-\frac{1}{d}\left(M+s_{0}+\hat{\rho}_{k}^{1}\right) \lambda^{2 k},
$$

where $\hat{\rho}_{k}^{1}=O\left(k \lambda^{k}\right)$ is some small coefficient and $\alpha=\frac{c x^{+}}{y^{-}}-1$ (see formula (11)).

As it follows from Lemma 1, the conservative non-orientable Hénon map $\bar{x}=y, \quad \bar{y}=$ $M+x-y^{2}$, where $M$ satisfies (14), is the normal (rescaled) form for the first return maps $T_{k}$ with all sufficiently large $k$. This Hénon map has no elliptic fixed points, however, there exists an elliptic 2-periodic orbit for $0<M<1$. Thus, we obtain, by (14), that the first return map $T_{k}$ has a fixed point with multipliers $\nu_{1}=+1, \nu_{2}=-1$ (i.e., when $M=0$ ) if

$$
\mu=\mu_{k}^{ \pm}=-\lambda^{k} y^{-} \alpha\left(1+k \beta_{1} \lambda^{k} x^{+} y^{-}\right)-\frac{1}{d}\left(s_{0}+\hat{\rho}_{k}\right) \lambda^{2 k},
$$

and a 2-periodic orbit with multipliers $\nu_{1}=\nu_{2}=-1$ (i.e., when $M=1$ ) if

$$
\mu=\mu_{k}^{2-}=-\lambda^{k} y^{-} \alpha\left(1+k \beta_{1} \lambda^{k} x^{+} y^{-}\right)-\frac{1}{d}\left(s_{0}+1+\hat{\rho}_{k}\right) \lambda^{2 k} .
$$

Thus, the first return map $T_{k}$ has in this case an elliptic 2-periodic orbit when $\mu \in \mathrm{e}_{k}^{2}$, where $\mathrm{e}_{k}^{2}$ is the interval of values of $\mu$ with border points $\mu=\mu_{k}^{ \pm}$and $\mu=\mu_{k}^{2-}$. Evidently, if $\alpha \neq 0$, the intervals $\mathrm{e}_{k}^{2}$ with sufficiently large and different $k$ do not intersect. 


\subsection{Proofs of Theorems 2 and 3 .}

Proof of Theorem Q By (28) and (29), the equations of the bifurcation curves $L_{k}^{2+}$ and $L_{k}^{2-}$, which are boundaries of the domain $E_{k}^{2}$, can be written as follows

$$
\begin{gathered}
L_{k}^{2+}: \quad \mu=-\lambda^{k} y^{-}\left(\frac{c x^{+}}{y^{-}}-1\right)\left(1+k \beta_{1} \lambda^{k} x^{+} y^{-}\right)-\frac{s_{0}+\cdots}{d} \lambda^{2 k}, \\
L_{k}^{2-}: \quad \mu-\lambda^{k} y^{-}\left(\frac{c x^{+}}{y^{-}}-1\right)\left(1+k \beta_{1} \lambda^{k} x^{+} y^{-}\right)-\frac{1+s_{0}+\cdots}{d} \lambda^{2 k} .
\end{gathered}
$$

Since $\lambda^{2 k} \ll \lambda^{k}$, the domains $E_{k}^{2}$ with sufficiently large $k$ do not mutually intersect and do not intersect the axis $\mu=0$, if $c x^{+} \neq y^{-}$. Thus, the domains do not always intersect in the cases with $c<0$. However, at the global resonance $\alpha=\left(c x^{+} / y^{-}-1\right)=0$ (which is possible only when $c>0$ ), as it follows from (30) and (31), all the domains $E_{k}^{2}$ with sufficiently large $k$ do mutually intersect and all of them intersect the axis $\mu=0$ (as in Figure 7).

Proof of Theorem 3 . Assume, for more definiteness, that $d>0$ for all the cases under consideration. The case $d<0$ is treated in the same way. Assume that $f_{0}$ satisfies conditions A, B and $\mathrm{D}$ and that the resonant condition $\alpha=0$ takes place for $f_{0}$. Then, for the one parameter family $f_{\mu}$ with fixed $\alpha=0$, the intervals $\mathrm{e}_{k}^{2}$ have, by (28)-(29), the form

$$
\mathrm{e}_{k}^{2}=\left(-1-s_{0},-s_{0}\right) \frac{\lambda^{2 k}}{d}
$$

Evidently, if $-1<s_{0}<0$, these intervals will be nested and containing $\mu=0$. This implies that the diffeomorphism $f_{0}$ has infinitely many double-round elliptic periodic orbits.

As follows from Lemma 1, all the first return maps $T_{k}$ (with sufficiently large $k$ ) are reduced to the same rescaled normal form - the non-orientable Hénon map $\bar{x}=y, \bar{y}=-s_{0}+x-y^{2}$. It is well known that, for $-1<s_{0}<0$, the elliptic 2-periodic orbit of this map is generic if

$s_{0} \neq-\frac{1}{2} ;-\frac{3}{4} ;-\frac{5}{8}$. These exceptional cases are related, respectively, to the resonances $1: 4,1: 3$ and to an elliptic point (at $s_{0}=-\frac{5}{8}$ ) whose first Birkhoff coefficient is zero.

\section{Acknowledgements.}

The authors thank D.Turaev and L.Lerman for fruitful discussions and remarks.

\section{References}

[1] Gavrilov, N.K. and Shilnikov, L.P., On three-dimensional dynamical systems close to systems with a structurally unstable homoclinic curve. I, Math. USSR Sbornik, 1972, v. 17, pp. 467485; II, 1973, v. 19, pp. 139-156.

[2] Newhouse, S.E., Diffeomorphisms with infinitely many sinks. Topology, 1974, v. 13, pp. 9-18.

[3] Gonchenko, S.V., On stable periodic motions in systems close to a system with a nontransversal homoclinic curve. Russian Math. Notes, 1983, v. 33, No. 5, pp. 745-755.

[4] Newhouse, S.E., Quasi-elliptic periodic points in conservative dynamical systems. Amer. J. Math., 1977, v. 99, pp. 1061-1087. 
[5] Gonchenko, S.V., Shilnikov, L.P., and Turaev, D.V., Elliptic periodic orbits near a homoclinic tangency in four-dimensional symplectic maps and Hamiltonian systems with three degrees of freedom. Regul. Chaotic Dyn., 1998, v. 3, No. 4, pp. 3-26.

[6] Gonchenko, S.V., Shilnikov, L.P., and Turaev, D.V., Existence of infinitely many elliptic periodic orbits in four-dimensional symplectic maps with a homoclinic tangency. Proc. Steklov Inst. Math., 2004, v. 244, pp. 115-142.

[7] Mora, L. and Romero, N., Moser's invariant curves and homoclinic bifurcations. Dyn. Sys. and Appl., 1997, v. 6, 29-42.

[8] Gonchenko, S.V. and Gonchenko, M.S., On cascades of elliptic periodic points in twodimensional symplectic maps with homoclinic tangencies, Regul. Chaotic Dyn., 2009, v. 14, No. 1, pp. 116-136.

[9] Biragov, V.S., Bifurcations in a two-parameter family of conservative mappings that are close to the Henon map. Selecta Math.Sov., 1990, v. 9, pp. 273-282. [Originally publ. in Methods of qualitative theory of differential equations, Gorky State Univ., 1987, pp. 10-24.]

[10] Biragov, V.S. and Shilnikov, L.P., On the bifurcation of a saddle-focus separatrix loop in a three-dimensional conservative system. Selecta Math. Sov., 1992, v. 11, pp. 333-340. [Originally publ. in Methods of qualitative theory and theory of bifurcations, Gorky State University, 1989, pp. 25-34.]

[11] Gonchenko, S.V. and Shilnikov, L.P., On two-dimensional analytic area-preserving diffeomorphisms with infinitely many stable elliptic periodic points. Regul. Chaotic Dyn., 1997, v. 2, No. 3/4, pp. 106-123.

[12] Gonchenko, S.V. and Shilnikov, L.P., On two-dimensional area-preserving diffeomorphisms with infinitely many elliptic islands. J. Statist. Phys., 2000, v. 101, No. 1/2, pp. 321-356.

[13] Gonchenko, S.V. and Shilnikov, L.P., On two-dimensional area-preserving mappings with homoclinic tangencies. Doklady Mathematics, 2001, v. 63, No. 3, pp. 395-399.

[14] Gonchenko, S.V. and Shilnikov, L.P., On two-dimensional area-preserving maps with homoclinic tangencies that have infinitely many generic elliptic periodic points. J. Math. Sci., 2005, v. 128, No. 2, pp. 2767-2773. [Originally publ. in Notes of S.-Petersburg Steklov Math. Inst., 2003, v. 300, pp. 155-166.]

[15] Poincare, H., Les méthodes nouvelles de la mécanique céleste, Tome III, Gauthier-Villars, 1899.

[16] Pugh, C. and Robinson, C., The $C^{1}$ closing lemma, including Hamiltonians. Ergod. Th. Dynam. Sys., 1983, v. 3, pp. 261-313.

[17] Takens, F., Hamiltonian systems: generic properties of closed orbits and local perturbations. Math. Ann., 1970, v. 188, pp. 304-312.

[18] Takens, F., Homoclinic points in conservative systems. Invent. Math., 1972, v. 18, pp. 267292. 
[19] Rüssman, H., Kleine Nenner I, Über invariante Kurven differenzierbarer Abbildungen eines Kreisrings.- Nachr. Akad. Wiss. Gött., Math. Phys. Kl. II, 1970, pp. 67-105.

[20] Vasiliev, A.A., Neishtadt, A.I., Simó, C., and Treschev, D.V., Stability Islands in Domains of Separatrix Crossings in Slow-Fast Hamiltonian Systems. Proc. of the Steklov Inst. of Math., 2007, v. 259, pp. 236-247.

[21] Lamb, J.S.W. and Quispel, G.R.W., Reversible k-symmetries in dynamical systems. Physica D, 1994, v. 73 , No. 4, pp. 277-304.

[22] Dmitriev, A.S., Komlev, Yu.A., and Turaev D.V., Bifurcation phenomena in the 1:1 resonant horn for the forced van Der Pol-Duffing equation, Int. J. Bifurcation Chaos, 1992, v. 2, No. 1, pp. 93-100.

[23] Delshams, A., Gonchenko, S.V., Gonchenko, V.S., Lazaro, J.T., and Sten'kin, O.V., Abundance of attracting, repelling and elliptic orbits in two-dimensional reversible maps. Nonlinearity, 2013, v. 26, No. 1, pp. 1-35.

[24] Gonchenko, S.V. and Shilnikov L.P., Invariants of $\Omega$-conjugacy of diffeomorphisms with a structurally unstable homoclinic trajectory. Ukrainian Math.J., 1990, v. 42, No. 2, pp. 134140 .

[25] Gonchenko, S.V., Shilnikov, L.P., and Turaev, D.V., Homoclinic tangencies of arbitrarily high orders in conservative and dissipative two-dimensional maps. Nonlinearity, 2007, v. 20, pp. 241-275.

[26] Shilnikov, L.P., Shilnikov, A.L., Turaev, D.V., and Chua, L.O., Methods of qualitative theory in nonlinear dynamics, Part I. World Sci., Singapore, 1998.

[27] Moser, J., The analytic invariants of an area-preserving mapping near a hyperbolic fixed point. Comm. Pure Appl.Math., 1956, v. 9, pp. 673-692.

[28] Gonchenko, S.V. and Shilnikov L.P., Arithmetic properties of topological invariants of systems with a structurally unstable homoclinic trajectory. Ukrainian Math.J., 1987, v. 39, No. 1, pp. 21-28.

[29] Gonchenko, S.V., Sten'kin, O.V., and Turaev, D.V., Complexity of homoclinic bifurcations and $\Omega$-moduli. Int. J. Bifurcation and Chaos, 1996, v. 6, No. 6, pp. 969-989.

[30] Turaev, D. and Rom-Kedar, V., Elliptic islands appearing in near-ergodic flows. Nonlinearity, 1998, v. 11, No. 3, pp. 575-600.

[31] Devaney, R. and Nitecki, Z., Shift automorphisms in the Hénon mapping. Comm. Math. Phys., 1979, v. 67, pp. 137-146.

[32] Afraimovich, V.S. and Shilnikov, L.P., Quasiattractors. Nonlinear Dynamics and Turbulence, eds G.I.Barenblatt, G.Iooss, D.D.Joseph (Boston,Pitmen), 1983, pp. 1-34. 